\title{
METASTATIC OSTEOCHONDROMA OF MAXILLA FROM PRIMARY TUMOR OF TIBIA
}

REPORT OF CASE

John W. Kemper, D.D.S., M.D.,* and Herbert J. Bloom, D.D.S., M.S. $\dagger$

S ARCOMAS are malignant neoplasms of connective tissue origin. They may $N$ be so-called pure, that is, connective tissue growths resembling an adult structure, or a considerable degree of differentiation may be present with intercellular substance that is clearly fascia, cartilage, bone, etc. Descriptive terms, such as fibrosarcoma, chondrosarcoma, osteosarcoma (or osteogenic sarcoma), have therefore come into being. The existence of more than one parent tissue in these tumors is depicted by terms such as osteochondrosarcoma.

Osteogenic sarcoma is considered to be one of the most common of malignant bone tumors. It is also regarded as one of the most malignant and incurable of all growths. It is scen more frequently in children and young adults, becoming increasingly rare in middle life. A clinical history of trauma is not uncommon, followed by the appearance of a sharply demareated, painful swelling at the site of the injury. It usually occurs at the end of the shaft of the long bones, principally in the region of the knee. It occasionally occurs as a primary lesion in the jawbones.

Early rapid growth is marked by discomfort and often an elevated temperature mimicking the picture of acute infection. The prognosis in almost all osteogenic sarcomas is very grave, and the younger the patient the worse the outlook and the more rapid the course.

The tumor grows expansively, but also infiltrates adjacent tissue, the cells creeping along fascial planes, between muscle fibers, and through Haversian canals, thus making successful surgical attempts unlikely and recurrence and metastasis common. It forms a bulky, highly vascular mass with vessels that are young and thin-walled, so that invasion by tumor cells takes place readily and metastasis occurs early by the hematogenous route-notably to the lungs. Indeed, the lungs may well be invaded before a diagnosis of the primary tumor can be made, and radiographic examination of the lungs should be made for metastasis in all cases before surgical treatment is considered. Should tumor emboli pass through the pulmonary capillaries, they may lodge in any organ; however, metastasis of osteogenic sarcoma to other bones is rather rare.

From the Department of Oral Surgery, University Hospital, University of Michigan.

* Professor of Oral Surgery and Head of the Department of Oral Surgery, School of Dentistry and University Hospital, University of Michigan.

†Instructor in Postgraduate Oral Surgery, W. K. Kellogg Institute of Graduate and Postgraduate Dentistry. 


\section{CASE REPOR'T}

History - - T. A., No. 455150, a 13-year-old white girl, was first admitted to the Bone and Joint Service of the University Hospital, in January, 1940. Approximately five months prior to this date she had tripped and fallen on the right knee. The abrasions healed rapidly, but two months later she noticed pain in the area sufficient to interfere with function. Swelling followed shortly, and the signs and symptoms gradually increased in severity. Her past and familial history were entirely irrelevant and noncontributory.

Physical Examination.-The patient was a well-developed, well-nourished white girl, appearing chronically ill. Examination of the extremities revealed some stiffening of the right knee with a tender, inflammatory swelling about the joint. The temperature was $99.2^{\circ} \mathrm{F}$. Further careful and detailed physical examination and laboratory studies failed to reveal any other significant findings.

Radiographic Examination.--Examination of both upper tibiae revealed a marked bone sclerosis of the proximal portion of the right tibial shaft. The radiologists considered the possibility of a sclerosing osteomyelitis, but regarded the probability of a primary bone tumor. Routine radiographic examination of the chest was entirely negative.

Course.-As a definite diagnosis could not be established by the usual clinical and radiographic methods, the lesion was biopsied. A diagnosis of spindle-cell osteochondrosarcoma was returned and, in view of lack of evidence of metastases, amputation was recommended. Accordingly, the right leg was removed by the orthopedic surgeons at the junction of the middle and lower thirds of the femur. Convalescence was uneventful and the wound healed without complication. The patient was discharged on the twenty-third postoperative day with instructions to return for postoperative examinations.

Subsequent Course.-For a period of almost two years after hospital discharge the patient was entirely well. Periodic examinations were negative for recurrence or metastases. In March, 1942, she again returned to the hospital, stating that a few months previous there had been an insidious onset of pain over the lateral aspect of the left femoral condyle, with the complaint that the pain had gradually increased in severity, and recently there had been swelling in this region.

Examination revealed a firm, smooth, symmetrical tumor approximately 6 by $7.5 \mathrm{~cm}$. in size, situated just above the left lateral femoral condyle. Further routine examination and accumulated laboratory data were essentially normal. Chest films remained negative. A biopsy of the left leg tumor disclosed an osteochondrosarcoma of the same general type as that of the primary neoplasm, but less differentiated. During this admission a left mid-thigh amputation was performed. The immediate, as well as the complete, postoperative course was uneventful.

In August, 1942, five months after the last discharge date, the patient noted pain and slight swelling in the left maxillary mucobuccal fornix. Her local dentist found the maxillary left first and second molars to be tender to 
percussion and moderately mobile, and removed these teeth. Temporary relief was obtained, but two weeks later a definite mass was visible at the recent operative site.

One month after the extractions the patient was seen in the Department of Oral Surgery of the University Hospital. Examination revealed marked facial asymmetry with definite prominence over the left cheek. A firm, nonfluctuant, tender mass could be readily outlined over the left alveolar ridge and anterior wall of the antrum. Intraorally, a fleshy tumor extended to the mesial aspect of the first premolar and posteriorly to the maxillary tuberosity. The adjacent dentition was partially covered, the mucobuceal fornix obliterated, and the palatal mucoperiosteum expanded toward the midline. A large, firm abdominal mass in the right upper quadrant was considered to be due to liver metastases.

Radiographic examination of the paranasal sinuses demonstrated an extensive neoplasm involving the left maxillary and ethmoid sinuses, left alveolar ridge, and soft tissues of the left cheek. There was also radiographic evidence of recurrent neoplasm at the distal end of the left femur. A seont film of the abdomen showed intra-abdominal calcification, and metastases of the osteochondrosarcoma were considered a likelihood.

By biopsy the intraoral mass was found to be identical with that of the previously occurring tumors of the extremities.

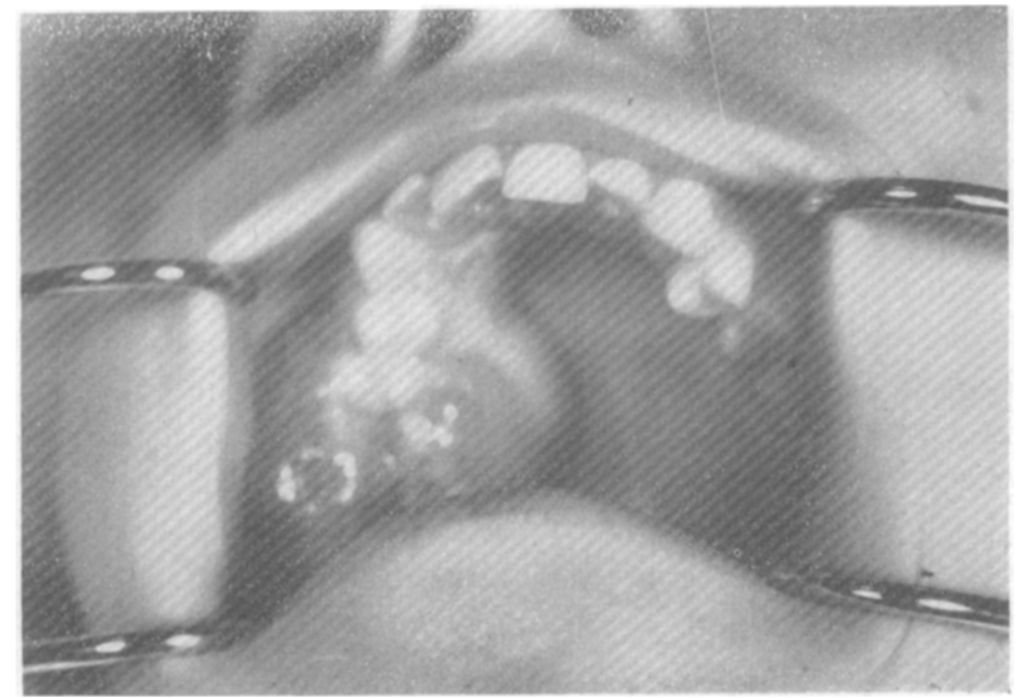

Fig. 1.-Metastatic osteochondroma of maxilla one month following extraction of teeth.

Because of the extensive invasion and unquestioned widespread metastases, therapeutic measures were necessarily confined to symptomatic treatment. An attempt to reduce the size of the maxillary mass by radiation therapy failed, and it was decided that surgical intervention to facilitate mastication and promote a feeling of well-being was in order. Under averlin antesthesia the oral portion of the mass was excised by cautery.

A relatively short period of relief was followed by progressive growth, and in January, 1943, the patient was again admitted for a second partial cautery excision of the maxillary neoplasm. At this time she complained of nasal ob- 